Akzeptiertes Manuskript in: Karidi, M. u.a. (Hg.): Resilienz. Wiesbaden: Springer 2018, 203-225

Abstract

Nach einer Einstimmung wird im zweiten Kapitel Resilienz in interdisziplinären Diskursen gesichtet, um resilienzsensible Bildung als Stärkung von Menschen und Systemen in der Außeinandersetzung mit Inhalten herauszuarbeiten. Das dritte Kapitel ordnet und entfaltet resilienzsensible Bildung in der Vielfalt möglicher Verständnisse, um Resilienz als Response-Strategie bildend zu profilieren und exemplarisch anhand der Methode „Professionelle Simulation“ zu profilieren. Im vierten Kapitel wird der Ertrag gebündelt und das Potential resilienzsensibler Bildung aufgezeigt.

Stichwörter: Resilienzsensible Bildung, Response-Strategie, Professionelle Simulation, Habitus

Manfred Riegger

\title{
Resilienzsensible Bildung
}

Resilienz als Response-Strategie durch Professionelle Simulation (ProfiS) entwickeln

Der Aufbau dieses Beitrages sieht folgendermaßen aus: Nachdem an zwei Fallbeispielen resilienzsensible Bildung konkretisiert wird, erfolgt im zweiten Kapitel eine systematische Verschränkung von Bildung und Resilienz im interdisziplinären Diskurs, um die spezifische Anschlussfähigkeit aufzuzeigen. Diese Ergebnisse werden im dritten Kapitel weiterentwickelt, indem Resilienz als Response-Strategie entfaltet und im methodischen Vorgehen der Professionellen Simulation verdeutlicht wird. Abschließend wird das Potential resilienzsensibler Bildung aufgewiesen.

\section{Einstimmung: Bilder und Fälle}

Resilienz wird im Bild häufig in Szene gesetzt als karge, lebensfeindliche Landschaft, in der einsam ein kleines Pflänzchen wächst, gelegentlich mit Blüte. Meint Resilienz also irgendein Leben gegen alle Wahrscheinlichkeit inmitten einer lebensfeindlichen Umgebung? Pflanzen mögen wachsen, aber sie handeln nicht, weshalb wohl auch das Bild des „Stehaufmännchens“ zu finden ist. Ein solches Bild suggeriert einfach Nehmerqualitäten. Doch meint Resilienz v.a. die Fähigkeit der Hinnahme und Bewältigung von dem, was Menschen zustößt? Beide Bilder erscheinen im Blick auf 
Akzeptiertes Manuskript in: Karidi, M. u.a. (Hg.): Resilienz. Wiesbaden: Springer 2018, 203-225

Bildung stark unterkomplex, weshalb ich zunächst zwei Fallbeispiele resilienzsensibler Bildung mittels einer Professionellen Simulation (vgl. Heil und Riegger 2017) skizzieren und die beiden Bilder anreichern werde. In Klammern sind die Phasen der Simulation angegeben (vgl. Riegger und Heil 2017b, S. 126), anhand derer unten die Verlaufsstruktur resilienzsensibel gestalteter Bildungsprozesse beschrieben wird (vgl. 3.2.3):

Zunächst sind wir im Ethikunterricht einer bayerischen Förderschule. Anwesend sind vier 14jährige Schüler (vgl. Kuhn 2013, S. 46): Die Gruppe sitzt mit der Lehrerin zusammen im Stuhlkreis. In der Mitte liegen weiße DIN-A4-Blätter. Die Lehrperson beginnt: „Auf diesen Blättern könnten Bilder zu sehen sein. Besondere Bilder. Es sind alles Frühstücksbilder, auf denen auch Du zu sehen bist.“" (ebd.) (=Szenendesign) Die Leitung stimmt auf die Simulation ein: „Wir stellen uns ein Bild vor. Und zwar sieht sich jeder in der Situation, in der er frühstückt.“" (ebd.) (=Einstimmung) Zu den Schülern gewandt sagt dann die Simulationsleitung: „Jeder nimmt sich nun ein Blatt und stellt diese Situation dar. Ihr dürft malen oder schreiben.“ (ebd.) (=Impuls der Leitung) „Die Schüler nehmen ein Blatt und Stifte und gestalten, an einem frei gewählten Ort im Klassenzimmer, ihr Frühstücks-Bild.“ (ebd.) (=Handlungen durchführen) Zurück im Sitzkreis stellen alle „nacheinander ihr Frühstückserlebnis vor. Hierbei wird zuerst das Bild beschrieben und dann das dazugehörige Erlebnis erzählt. Die Schüler hören interessiert zu. [...] Drei Schüler schildern eine Frühstückssituation an einem großen, reichhaltig gedeckten Tisch, an dem sie mit der ganzen Familie sitzen. Es gibt viele Leckereien, man hat Zeit, man unterhält sich. Verschiedene Nahrungsmittel werden aufgezählt, die auf gar keinen Fall fehlen dürfen: Nutella, Cornflakes, Eier, Croissants, ... Vor allem eher ungesundes Essen steht auf dem Speiseplan. Ein Schüler erzählt, dass bei ihnen immer das Fernsehgerät laufe, was völlig uncool sei, da man sich nicht richtig unterhalten könne und eigentlich jeder für sich alleine frühstücke. Deshalb nähme er häufig seinen Teller und gehe zum Frühstücken in sein Zimmer: ,Da ist es leise. Da habe ich meine Ruhe.' Zuletzt beschreibt die Lehrkraft ihre Frühstückssituation, welche im Rahmen der Familie stattfindet. $\mathrm{Zu}$ essen gibt es bei ihr ein gesundes Frühstück, bestehend aus Haferflocken, Obst und Naturjoghurt." (ebd., S. 46f) (=Kommunikation über eigene Erlebnisse) Die Beschreibung der Lehrkraft bleibt „nicht unkommentiert: ,Boah, das ist ja voll gesund!' oder ,Und das schmeckt? und ,Das dauert ja voll lange, bis Sie das zubereitet haben. Wann stehen Sie denn 
Akzeptiertes Manuskript in: Karidi, M. u.a. (Hg.): Resilienz. Wiesbaden: Springer 2018, 203-225

morgens auf?““ (ebd., S. 47) (=Perspektivenwechsel) Auf all diese Fragen erhalten die Schüler zu einem späteren Zeitpunkt eine Antwort, denn die Lehrkraft stellt fest, dass es sich bei allen vier Schülererlebnissen „um Wochenend- bzw. Ferienerlebnisse handeln muss“ [...]. „Daher fragt sie nach, ob dies so sei und ob sich das allägliche Frühstückserlebnis von dem eben beschriebenen unterscheide. [...] In einer zweiten Runde erzählen die Schüler, wie sie das Frühstück im Alltag, morgens vor der Schule, erleben. [...] S1: ,Ich frühstücke alleine, esse wenig und schnell, da das keinen Spaß macht.' S2: ,Wenn ich aufstehe, sitzen meine Eltern vor dem Fernseher. Oft frühstücke ich dann gar nicht, weil mich das nervt und ich schnell weg möchte.' S3: ,Ich frühstücke nichts, weil ich immer zu spät aufstehe und keiner mehr zuhause ist. Ich kaufe manchmal etwas unterwegs beim Bäcker oder esse gar nichts.' S4: ,Ich frühstücke zusammen mit meinem Bruder, der muss auch in die Schule.““ (ebd.) Mit folgender Aufforderung wird die Simulation beendet: „'Jetzt dürft Ihr entscheiden, ob Ihr euer Bild behalten möchtet oder ob Ihr es dalassen möchtet.' Alle Schüler nehmen Ihr Bild und sortieren es in den Ethik-Hefter ein." (ebd.) (=Beendigung der Simulation) In der anschließenden Reflexion über die Erlebnisse redet man darüber, wie schön es ist zusammen zu frühstücken, welche gesunden Nahrungsmittel schmecken könnten. „Entsprechende Nahrungsmittel, wie Vollkornbrötchen und Bioprodukte (Butter, Marmelade, Wurst, Käse), sind vorhanden, welche nun die Grundlage für das gemeinsame Frühstück darstellen. Zusammen wird der Tisch gedeckt. Es herrscht eine absolut friedliche, fast schon familiäre Atmosphäre. Die Schüler erzählen von ihren Ferienerlebnissen, bemerken aber nebenbei, dass Bioprodukte besser schmecken, als das, was sie normalerweise essen. Ihr Interesse an einem gesunden Frühstück steigt, sodass die Lehrkraft anbietet, für die nächste Stunde ein Haferflockenmüsli mit Obst vorzubereiten. Ein Schüler ist immer noch sehr skeptisch: ,Ob das wohl schmeckt?"“ (ebd.)

Das zweite Beispiel einer Professionellen Simulation entstammt der Lehrerbildung an der Universität Augsburg (vgl. ausführlich Riegger und Heil 2017b, S. 135ff.), in welcher Unterrichtsstörungen simulierend thematisiert wurden. Globale Bildungsziele werden in Bezug auf folgende Intention bzw. Kompetenzerwartung konkretisiert: Die schulische Konfliktsituation: „Eine Schülerin verweigert im Religionsunterricht die Mitarbeit" wird mittels Professioneller Simulation wahrgenommen und gezielt gestaltet. 
Akzeptiertes Manuskript in: Karidi, M. u.a. (Hg.): Resilienz. Wiesbaden: Springer 2018, 203-225

Im vorbereitenden Gespräch werden Informationen über den zu simulierenden Konfliktfall im Unterricht gesammelt und gebündelt. Danach wird im Seminarraum der Universität ein Tisch aufgestellt. Dahinter nimmt die eingeladene Lehrkraft Platz. Sie simuliert Mareike (alle Namen wurden geändert), eine Schülerin aus ihrer Klasse. Ein Buch und ein Blatt liegen vor ihr. Im Halbkreis gegenüber sitzen die Studierenden. (=Szenendesign) Die Leitung beginnt: „Wir sind im Zimmer der 8. Klasse an einer Mittelschule (= Hauptschule mit der Möglichkeit eines Mittleren Schulabschlusses) in Bayern. Der Unterricht ist in vollem Gange. Die Schülerinnen und Schüler lesen im Religionsbuch. Der Lehrer, Herr Meier, ruft die einzelnen Schüler auf. Mareike ist an der Reihe." (=Einstimmung durch die Leitung) Zu den Studierenden gewandt sagt dann die Simulations- und Seminarleitung: „Sie können nun die Rolle von Herrn Meier übernehmen. Sie entscheiden selbst, wann Sie in die Szene treten und beginnen selbstständig." (=Impuls der Leitung) Der Simulationsprozess (=Handlungen durchführen) beginnt: Ein Studierender steht auf und übernimmt die Lehrerrolle. Mareike schaut aus dem Fenster. Herr Meier zögert und sagt: „Mareike, liest du bitte den Text!“ „Herr Meier, ich will nicht!“, bekommt er zur Antwort. Nach kurzer Zeit fragt er: „Mareike, warum willst du nicht lesen?“ „Ich habe keine Lust!“, ist zu vernehmen und der Lehrer entgegnet: „Das kann ich verstehen, dass man manchmal keine Lust hat. Willst du etwas anderes tun?“ „Ja, rausgehen“, und schon ist Mareike auf dem Weg zur Tür. Daraufhin bricht der Student die Simulation ab. Nach weiteren Simulationen lässt man die Szenerie noch aufgebaut, setzt sich aber im Stuhlkreis zusammen und reflektiert über die Simulationen der Reihe nach. Zunächst erzählt der simulierende Studierende 1 (=Kommunikation über eigene Erlebnisse): „Ich wollte Mareike Verständnis entgegenbringen und hoffte, dass sie dann liest.“ ... Die Lehrkraft in der Schülerinnenrolle erzählt: „Ich dachte: ,Toll, jetzt kann ich machen, was ich will!“ Daraufhin ergänzen die Beobachtenden (=Perspektivenwechsel): „Ich als Mareike empfand ein Gefühl des Sieges.“ „Als Herr Meier ging es mir schlecht, weil ich dachte, dass ich für immer die Autorität vor den anderen Schülern verloren habe." Konnten sich alle äußern, beendet die Leitung die Simulation, indem die Szenerie abgebaut und die gewohnte Sitzordnung eingenommen wird (=Beendigung der Simulation). Die habitusbezogene Reflexion erfolgt mindestens in zwei Varianten: Zuerst bindet die Lehrperson die Simulation an ihren Habitus zurück, indem sie über ähnliche Anforderungssituationen aus ihrem Schulalltag spricht und die Studierenden knüpfen an ihren Habitus an, indem sie auf 
Akzeptiertes Manuskript in: Karidi, M. u.a. (Hg.): Resilienz. Wiesbaden: Springer 2018, 203-225

den Punkt bringen, was sie handlungspraktisch gelernt haben (=pragmatischreflexive Reflexion). Sodann werden gemeinsam Bezüge zu unterschiedlichen wissenschaftlichen Theorien erörtert und wissenschaftlich begründete Deutungen von Konflikten und Konfliktregelungen erarbeitet (=reflexiv-wissenschaftliche Reflexion). Abschließend werden die Wirkungen der Simulation eruiert (=Evaluation). Eine Studierende antwortete dabei: „Eigentlich war es doch nur ein Spiel, aber ich fühlte mich wie in der Schule. Ich dachte nicht nur theoretisch über Unterrichtsstörungen nach, sondern probierte unterschiedliche Handlungsmöglichkeiten aus. Das war ungewohnt, aber auch sehr hilfreich.“

Diese beiden Fallbeispiele aus dem schulischen und hochschulischen Allag vermitteln zunächst einen ersten Eindruck von resilienzsensibler Bildungspraxis, die im Folgenden wissenschaftlich eingeholt wird.

Zuvor aber noch zur Weiterentwicklung der beiden Bilder: Resilienzsensible Bildung zielt im ersten Fallbeispiel durch das Entdecken und Anbieten von Frühstücksalternativen darauf $a b$, dass die Lernenden sich gegen die „Frühstücksöde“ (Bild 1) zuhause durchsetzt und damit quasi neu und vielfältigt erblühen. Vom zweiten Fallbeispiel her ist das zweite Bild zu ergänzen: Die Lehramtsstudierenden sind nicht auf einen Standpunkt bei Unterrichtsstörungen festgelegt, sondern können - wie ein Boxer - tänzeln. Noch besser: Sie können auch aus dem Kampf, aus dem Ring und dem ganzen Bild des Kampfes aussteigen und neue Wege gehen. Das Suchen und Finden alternativer Wege stehen bei resilienzsensibler Bildung im Mittelpunkt.

\section{Pädagogik, Religionspädagogik und Resilienz in interdisziplinären Diskursen}

Auf der Grundlage einer Standortbestimmung von Religionspädagogik (2.1) werden mögliche wissenschaftliche Zugänge im Blick auf Resilienz eröffnet (2.2), um innerhalb bildungstheoretischer Überlegungen (2.3) Resilienz in spezifischen Handlungsfeldern zu sichten. Folgender Kernbegriff von Resilienz fungiert als regulativer Suchbegriff:

Resilienz vom Lateinischen re-silire (dt. = zurückspringen) bzw. vom Englischen resilience (dt. = Spannkraft, Widerstandsfähigkeit, Elastizität) kann - in 
Akzeptiertes Manuskript in: Karidi, M. u.a. (Hg.): Resilienz. Wiesbaden: Springer 2018, 203-225

Erweiterung eines psychischen Verständnisses (Wustmann Seiler und Fthenakis 2016, S. 18) - im Kern verstanden werden als die Fähigkeiten und Prozesse von Personen und Systemen, erfolgreich mit belastenden und bedrohenden Umständen umzugehen (vgl. Vogt und Schneider 2016b, S. 181).

\subsection{Verständnis von Religionspädagogik bzw. -didaktik}

In der Religionspädagogik beschreibt, analysiert und reflektiert man religiöse Bildungsprozesse (vgl. Sajak 2012, S. 65) und bezieht sich in Forschung und Lehre „auf alle Bereiche der religiösen Erziehung, Bildung, Entwicklung und Sozialisation in Schule, Kirche (Religionsgemeinschaften) und Gesellschaft beziehungsweise in globalen Zusammenhängen“ (Schweitzer 2015, 1). Innerhalb dieses Verständnisses von Religionspädagogik verhilft sich Religionsdidaktik zu einer „wissenschaftlich begründeten reflexiven Kompetenz hinsichtlich der Planung, Durchführung und Evaluation religiöser Lernprozesse sowie deren edukativer Verantwortung“ (Ziebertz 2015, S. 20).

Um Verständigungsschwierigkeiten gegenüber nichttheologischen Wissenschaften in unserem Fall nicht zu provozieren, beziehen sich die beiden eingangs angeführten Fallbeispiele vorwiegend auf für alle Disziplinen akzeptable, innerweltliche Aspekte wie Frühstück und Unterrichtsstörungen.

\subsection{Wissenschaftliche Zugänge}

Die unterschiedlichen Wissenschaften versuchen auf unterschiedliche Art und Weise über Resilienz Aussagen zu machen. Grundsätzlich scheinen drei Zugänge entscheidend: empirische, normative und operative.

\subsubsection{Empirische Zugänge}

Empirische Untersuchungen (vgl. Gennerich und Riegel 2015, 1) zu Resilienz erheben Resilienz fördernde Faktoren. Geklärt werden soll, wie ein hohes Maß an Resilienz erreicht werden kann und welche Faktoren entscheidend sind: psychische, soziale, institutionelle, ökonomische, ökologische, politische oder gesellschaftliche, religiös-spirituelle u.v.a.m.

Verwendet werden unterschiedlichste Erhebungsmethoden: Resilienz als psychische Widerstandsfähigkeit wird beispielsweise mit der deutschsprachigen Version der auf G. M. Wagnild und H. M. Young (1993) zurückgehenden Resilienzskala (RS-25 und 
Akzeptiertes Manuskript in: Karidi, M. u.a. (Hg.): Resilienz. Wiesbaden: Springer 2018, 203-225

RS-11) ebenso gemessen (vgl. Schumacher et. al. 2005, Leppert et. al. 2008), wie mit der Connor-Davidson Resilience Scale (CD-RISC) (vgl. Sarubin et. al. 2015). Letztere verwenden Nguyen u.a. (2015) und verbinden sie mit einer Skala zum Gottesbild. Die RS-11 verwendet E. Stangl und trianguliert sie mit qualitativen Leitfadeninterviews (2016, S. 229-258). Insgesamt sind neben quantitativen Erhebungen auch qualitative auszumachen, wie die Pionierstudien zu Beginn der Resilienzforschung von Emmy E. Werner et. al. (vgl. 1971, 1982), die auch religiöse Überzeugungen einschlossen. Die qualitativen Vorgehensweisen untersuchen nicht selten vornehmlich personale bzw. individuelle Resilienz (vgl. z.B. Göppel und Zander 2017). Diese individuelle Sicht ist sicher mit einer sozialen zu verschränken (Sedmak 2016, S. 242), wozu auch sozioökonomische Aspekte zählen und ggf. geophysische ergänzt werden müssen (vgl. Vogt und Schneider 2016b, S. 184-187). Festzuhalten ist: Die Diskurse über individuelle und soziale Resilienz werden ähnlich geführt, nämlich mit einem Interesse an Schutz- und Risikofaktoren (vgl. Sedmak 2016, S. 238), weshalb nachfolgend normative Ansprüche thematisiert werden müssen. Da Bildungsprozesse von Subjekten her thematisiert werden, kann mit allgemein vorgegebenen Faktoren nicht gearbeitet werden.

\subsubsection{Normative Zugänge}

Thematisiert man Resilienz, so thematisiert man immer - mehr oder weniger explizit - auch das, was sein oder nicht sein soll. Diese Aussagen sind normative Setzungen (vgl. z.B. Nida-Rümelin und Gutmann 2016, S. 257) und können nicht durch Beobachtungen als wahr oder falsch eingestuft werden.

Wer von Resilienz spricht, verspricht - mehr oder weniger deutlich - „robust und flexibel auf die Umbrüche reagieren zu können“ (Vogt und Schneider 2016b, S. 180). Selbst wenn diese positive Erwartung mit religiösen Überzeugungen umgedeutet wird, indem selbst einem Opfer etwas Positives abgewonnen werden kann (vgl. die Bedeutungsunterschiede von victim und sacrifice) und die Schwäche zur Stärke wird (vgl. Vogt 2009), bleibt Normativität leitend, nur unter verändertem Vorzeichen. Die skizzierten religiösen Überzeugungen (vgl. Schneider und Vogt 2016) scheinen neben profanen, rein innerweltlichen Überzeugungen für Resilienz nicht unwesentlich zu sein. Festzuhalten ist: Ein rein empirischer Diskurs über Resilienz ist nicht möglich und Bildungsintentionen bzw. Kompetenzen müssen begründet werden. 
Akzeptiertes Manuskript in: Karidi, M. u.a. (Hg.): Resilienz. Wiesbaden: Springer 2018, 203-225

\subsubsection{Operative Zugänge}

Religionspädagogik kann auch einen operativen Zugang thematisieren (vgl. Gennerich und Riegel 2015, 3). Gefragt wird: Wie müssen Bildungsprozesse strukturiert und rhythmisiert sein, damit diese begleitet und gefördert werden können? Entwickelt werden Theorien von Bildungspraxis für Bildungsprozesse, ohne schon gewissermaßen die Praxis selbst im Sinne einer „Anwendungswissenschaft“ zu sein, weshalb hier eingangs zwei Fälle exemplarisch skizziert wurden.

Festzuhalten ist: Es geht weder um Idealbeispiele noch um Ratgeberliteratur, sondern um die didaktisch-methodischen Gestaltungsmöglichkeiten und ggf. um die empirische Untersuchung resilienzsensibler Bildungsprozesse. $\mathrm{Zu}$ nennen ist beispielsweise interventionsbezogene Resilienzforschung, d.h. Forschung, die auf die positive Beeinflussung von Resilienz zielt, wie sie Fritz Oser et. al. für den Übergang zwischen Schule und Lehre durchführte (vgl. 2004). In diesem Zusammenhang sind konkrete Lehr-Lernmethoden für resilienzsensible Bildungsprozesse passgenau und theoretisch begründet zu reflektieren, wie es hier ansatzhaft für die Professionelle Simulation erfolgt.

\subsection{Bildungstheoretische Zugänge}

\subsubsection{Grundlegung: bildungstheoretisches Verständnis}

In Anlehnung an Wilhelm von Humboldt kann Bildung - jenseits aller Unterschiede verstanden werden als die "differenzierte, gedanklich und sprachlich vermittelte Auseinandersetzung von Menschen mit sich, mit anderen und mit der Welt" (Dörpinghaus et. al. 2012, S. 10). Allgemeine Bildung schließt damit „alle unterschiedlichen Formen des Weltverstehens und des Weltumgangs“ (Dressel 2017, 1) ein und gilt als der Prozess, „in dem Individualität und vielgestaltige Weltbezüge wechselseitig vermittelt werden“ (ebd.). Grundlegend ist also ein medialer Prozess der Vermittlung, nicht im Sinne einer Weitergabe vorher festgelegter Inhalte, sondern im Menschen, zwischen Menschen und der Welt, der auch zwischen Subjekten und Inhalten vermittelt. Allem zu Grunde liegt - im religiösen Verständnis - Gott, weshalb der Prozess der Vermittlung mit, in und durch Gott als vorgängig erachtet wird (vgl. Boschki 2011, S. 84), ohne dadurch das allgemeine Bildungsverständnis zu beeinträchtigen. 
Akzeptiertes Manuskript in: Karidi, M. u.a. (Hg.): Resilienz. Wiesbaden: Springer 2018, 203-225

Als wesentlich für Bildung wird ein Paradox erachtet: Bildung kann „nur dann funktional sein [...], wenn sie nicht nur funktional ist“" (Dressel 2017, 1). Neben (angestrebten) Intentionen und Funktionen treten Unintendiertes und Nichtfunktionales. Dabei erweisen sich letztere möglicherweise als nachhaltiger und funktionaler als Intentionen und Funktionen (vgl. ebd.). Bildungserfolge sind „an der Erwartung zu messen, wie es gelingt, Menschen zu stärken, indem ihre Urteilskraft und Handlungsfähigkeit auf allen Feldern des Zusammenlebens entwickelt wird“ (ebd.). „Bildung ,stärkt die Menschen“, indem sie ,die Sachen klärt"“ (von Hentig, zitiert nach Dressel 2017, 1). Bildung ist dann resilienzsensibel, wenn sie nicht nur selbstoptimierend wirkt, sondern intentional in inhaltlicher Auseinandersetzung Menschen und Systeme stärkt, ohne Nichtfunktionales auszuschließen.

\subsubsection{Drei Prinzipien}

Für den religionspädagogischen Bildungsbegriff konstitutiv sind drei Prinzipien, die pädagogisch und theologisch begründet sind, innerhalb derer Resilienz verortet sein muss:

Bildsamkeit des Menschen: Pädagogisch besagt Bildsamkeit, dass „der Mensch keine inm von Natur vorgegebene Bestimmung hat, sondern sich in Wechselwirkung mit der Welt bilden und seine Bestimmung selbst hervorbringen muss" (Benner 2014, S. 7). Theologisch schließt hier der Begriff der Gottebenbildlichkeit an, in welchem sich das vorgängig von Gott bestimmte Menschsein ausdrückt, weshalb auf dieser Grundlage „der Mensch selbst- und weltbildend tätig werden und seine Bestimmung in Wechselwirkung mit der Welt suchen kann“ (ebd.). Beide Abhängigkeiten konvergieren so, „dass der Angewiesenheit der Religion auf menschliche Bildsamkeit eine Bildsamkeit des Menschen zur Religion korrespondiert, die aktiviert werden muss, wenn religiöse Erziehung gelingen soll“ (ebd., S. 27). Insofern entscheiden in resilienzsensiblen Bildungsprozessen Teilnehmende selbst, was aus ihrer Sicht für sie resilienzförderlich ist.

Aufforderung zur Selbsttätigkeit: Der Mensch ist in der pädagogischen Interaktion zur selbsttätigen Mitwirkung an seinem Bildungsprozess aufgefordert. Hier berühren wir die „pädagogische Grundparadoxie, dass Heranwachsende, die von ihrer Freiheit noch keinen durchweg vernünftigen Gebrauch machen können, gleichwohl im Prozess der pädagogischen Interaktion in ihrer Befähigung zum Selbstgebrauch der Freiheit wachsen können sollen“ (Dressel 2017, 3). Es geht um die 
Akzeptiertes Manuskript in: Karidi, M. u.a. (Hg.): Resilienz. Wiesbaden: Springer 2018, 203-225

„Fremdaufforderung zur Selbsttätigkeit“ (Dietrich Benner) oder wie mit Zwang Freiheit erreicht werden kann. Theologisch kann hier nach dem Verhältnis der pädagogisch-bildungstheoretischen und religiös-schöpfungstheologischen Erklärungen und Vergewisserungen gefragt werden, die nicht deduktiv und damit nicht-hierarchisch erfolgen (vgl. Benner 2014, S. 29). Bildungsprozesse müssen damit notwendig eine Fremdaufforderung zur eigenständigen Resilienzbildung enthalten, wie es in den beiden Fallbeispielen deutlich wird, da die Selbsttätigkeit der Teilnehmenden erst nach der Aufforderung durch die Leitung erfolgt.

Pädagogische Transformationen: Dieses Prinzip verweist darauf, dass neben individuellen Aufforderungen zur Selbsttätigkeit zugleich gesellschaftliche Einwirkungen zu berücksichtigen sind (vgl. ebd., S. 30). In den Blick kommt hier die Legitimität von Abstimmungsprozessen, die teils in den pädagogischen Situationen selbst und teils zwischen diesen und außerpädagogischen Situationen liegen. Abstimmungsprozesse „zwischen pädagogischen und außerpädagogischen Situationen und Kontexten lassen sich durch didaktische Transformationen von Weltinhalten zu Unterrichtsgegenständen oder durch gesellschaftspädagogische Transformationen bearbeiten, in denen außerpädagogische Handlungsfelder so umgestaltet werden“ (ebd.), dass Teilnehmende an ihnen partizipieren können. In resilienzsensiblen Bildungsprozessen müssen deshalb Gegebenheiten so in den Blick genommen werden, dass diese nicht nur individuell, sondern auch gesellschaftlich-politisch überstiegen, transformiert werden.

„In religionspädagogischen Kontexten treffen - wie in anderen Bereichspädagogiken auch - stets mehrere Transformationsaufgaben aufeinander“ (ebd., S. 31). Neben religiösen sind „immer auch ökonomische, ethische, pädagogische, politische und ästhetische Welterfahrungen zu berücksichtigen, ohne dass über die dabei zu klärenden Abstimmungsprobleme allein aus religiös-theologischen Perspektiven entschieden werden könnte“ (ebd.). Resilienzsensible Bildung muss sich damit „als kompatibel mit den Voraussetzungen einer pluralen Gesellschaft erweisen“ (Kumlehn 2015, 2.2), aber diese auch verändern wollen.

Nimmt man die genannten drei Prinzipien ernst, so darf Resilienz in Bildungsprozessen nicht nur als Persönlichkeitsbildung (vgl. dazu Riegger 2012) konzipiert werden, da die von den Teilnehmenden ausgehenden Bildungsprozesse innerhalb von unterschiedlichsten Systemen angesiedelt sind. Damit müssen resilienzsensible Bildungsprozesse nicht nur didaktische, sondern mindestens 
Akzeptiertes Manuskript in: Karidi, M. u.a. (Hg.): Resilienz. Wiesbaden: Springer 2018, 203-225

gesellschaftliche und ökologische Transformationsprozesse miteinbeziehen, ohne Zielentscheidungen zu vernachlässigen.

\subsubsection{Primat der Zielentscheidung}

Zielentscheidungen sind für resilienzsensible Bildung ausschlaggebend. Notwendig ist deshalb die dem Inhalt immanenten Ziele auf ihre fachdidaktische Bedeutsamkeit hin zu untersuchen und vom Bildungsbegriff her zu qualifizieren, um so zu Zielen für den konkreten Bildungsprozess zu kommen. Dieses „Primat der Zielentscheidung“ gegenüber der Inhaltlichkeit berücksichtigt ebenso das Primat der Zielentscheidung gegenüber methodischen Entscheidungen (vgl. Klafki 1991, S. 432). Aus (religions)pädagogisch-didaktischer Perspektive ist damit Resilienz in Bezug auf zielorientierte Inhalte ebenso unter die Lupe zu nehmen, wie bezüglich zielorientierter methodischer Vorgehensweisen. In anderen Worten: Inwiefern enthalten resilienzaffine Inhalte (z.B. Unterrichtsstörungen) immanente Ziele (z.B. Stärkung der Lehrperson oder Änderung der Verhältnisse) und inwiefern korrespondieren beide mit immanenten methodischen Strukturierungen von Bildungsprozessen (Implikationszusammenhang) wie der Professionellen Simulation?

\subsection{Resilienz in Handlungsfeldern der Bildung}

Mit Resilienz ist eine menschliche Fähigkeit angesprochen, die es schon immer gegeben haben mag, deren wissenschaftliche Erforschung aber gemeinhin mit dem Zwischenbericht der Kauai-Studie von Emmy Werner und Ruth Smith in Verbindung gebracht wird (vgl. Werner et. al. 1971, Werner und Smith 1982).

Seit über dreißig Jahren liegt das Hauptaugenmerk der bildungsrelevanten Resilienzforschung auf Kindern und Jugendlichen (vgl. Werner 2011, Zander 2011, Fröhlich-Gildhoff et. al. 2016, Wustmann Seiler und Fthenakis 2016). Dieser Schwerpunkt der Forschung erweitert sich zunehmend, beispielsweise in Richtung Erwachsene (vgl. Leipold 2015), sodass der ganze Lebenslauf in den Blick kommt (vgl. Rönnau-Böse, Fröhlich-Gildhoff Höfler 2016 und zum Ganzen z.B. Wink 2016). Ebenso wird Resilienz nicht nur in Beratung und Therapie (vgl. Welter-Enderlin und Hildenbrand 2016), sondern auch in unterschiedlichen Disziplinen und Kontexten des Sozialen untersucht (vgl. Endres und Maurer 2015) und mittlerweile zunehmend in der Theologie (vgl. Pohl-Patalong, Richter 2016, Richter und Blank 2016, Richter 2017), wobei sich neben Moraltheologie sowie Sozialethik (vgl. z.B. Sedmak 2013, 
Akzeptiertes Manuskript in: Karidi, M. u.a. (Hg.): Resilienz. Wiesbaden: Springer 2018, 203-225

Vogt und Schneider 2016a, Sautermeister 2016), Pastoralpsychologie (z.B. Stangl 2016), Seelsorge bzw. Diakonie (vgl. Hauschildt 2016) sowie Exegese (vgl. z.B. Gärtner 2016) und zurückhaltend die Religionsdidaktik (vgl. z.B. Oser 2013) an der Debatte beteiligen.

Darüber hinaus wird Resilienz zu Nachbardiskursen in Beziehung gesetzt und von ähnlichen Konstrukten wie Empowerment (vgl. Bucher und Domsgen 2016) abzugrenzen versucht. Beispielhaft seien hier genannt: Vulnerabilität bzw. Invulnerabilität und Robustheit (Hardiness) (vgl. Keul 2016), Salutogenese, die zentral mit dem Kohärenzgefühl arbeitet (vgl. Fröschl 2015, Nida-Rümelin und Gutmann 2016, S. 253f.), Coping (vgl. Stangl 2016, S. 100-103) und (Mit)Leidensfähigkeit als Empathie und Compassion (vgl. ebd. S. 115f.). Viele der angeführten Untersuchungen lassen zwar mehr oder weniger Bezüge zu bildungsrelevanten Aspekten erkennen, beschäftigen sich aber kaum mit der Planung und Gestaltung von Bildungsprozessen im engeren Sinne oder mit Resilienz als Response-Strategie. Diese beiden Aspekte werden im folgenden Kapitel bearbeitet.

\section{Responsive Resilienzbildung durch Professionelle Simulation}

Zunächst wird das Verständnis von Resilienz in und für Bildungsprozesse als Response-Strategie präzisiert, um es anschließend mit Professioneller Simulation zu operationalisieren.

\subsection{Vielfalt des Resilienzverständnisses in bildungstheoretischer Perspektive}

\subsubsection{Grundbedeutung}

Der bereits angeführte Kernbegriff von Resilienz, nämlich die Fähigkeiten und Prozesse von Personen und Systemen, erfolgreich mit belastenden und bedrohenden Umständen umzugehen (vgl. 2.) beinhaltet ein personales und ein soziales Verständnis von Resilienz. „Soziale Resilienz“ ist die „Fähigkeit von Gruppen und Gemeinschaften äußeren Stress und Beeinträchtigungen als Ergebnis von Veränderungen des Sozialen, Politischen und der Umwelt zu bewältigen“ (Adger 2000, S. 347, eigene Übersetzung).

Resilienz ist ein „dynamischer Prozess“ (Vogt und Schneider 2016b, S. 191) von Anpassung und Entwicklung (vgl. Rönnau-Böse und Fröhlich-Gildhoff 2015, S. 16), 
Akzeptiertes Manuskript in: Karidi, M. u.a. (Hg.): Resilienz. Wiesbaden: Springer 2018, 203-225

der sich in Interaktion zwischen Individuum und Umwelt (vgl. ebd.) bzw. im Spannungsfeld von Beharrung und Veränderung (vgl. Vogt und Schneider 2016b, S. 191) ereignet.

Resilienz ist eine „variable Größe“ (Rönnau-Böse und Fröhlich-Gildhoff 2015, S. 16), die sich im Laufe der Zeit verändert. Es ist ein „nicht klar steuerbarer, durch Unsicherheit wie erst noch zu gewinnendes Wissen gekennzeichneter Prozess“ (Endreß und Rampp 2015, S. 38).

Nicht zuletzt ist Resilienz „situationsspezifisch und multidimensional" (ebd.) und damit kein allgemeingültiges und universales Phänomen, sondern „bereichsspezifisch“ (ebd.), d.h. sie kann sich in verschiedenen Bereichen unterscheiden.

Die inhaltliche Variabilität und Dynamik des Resilienzverständnisses konvergiert mit der inhaltlichen Variabilität und Dynamik der Methode der Professionellen Simulation. Zudem implizieren Methode wie Inhalte die Mündigkeit von Subjekten intentional, weil Subjekte sich sowohl gegen Stärkungen entscheiden, als auch gesellschaftliche Transformationen anstoßen können. Diese globalen Begründungen im Sinne des Implikationszusammenhangs sind zu konkretisieren, indem auch einige der angesprochenen Aspekte im Folgenden für den Bildungsbereich zu spezifizieren sind, bevor Resilienz operationalisiert wird.

\subsubsection{Bereichsspezifische Resilienz}

Bildend kann Resilienz nur innerhalb unterschiedlicher Bereiche der Praxis thematisch werden. Benner unterscheidet hier sechs bildungsrelevante Praxisbereiche: „Politik“, „Kunst“, „Religion“, „Arbeit“, „Ethik“ und „Pädagogik“ (2014, S. 17). Diesen Bereichen entsprechend, kann Resilienz nur in bereichsspezifischen Handlungen und Urteilen grundgelegt werden, beispielsweise in personalen, sozialen und politischen, religiös-spirituellen und kulturellen, ökonomischen und ökologischen Bereichen. Die Handlungsfelder zeichnen sich durch unterschiedliche Handlungslogiken aus, stehen aber untereinander in Beziehung (vgl. ebd.). Treten Konflikte zwischen unterschiedlichen, resilienzaffinen Handlungslogiken auf, so sind sie bildend zu bearbeiten, indem Antworten, die einer einzigen Handlungslogik folgen, problematisiert, Kollisionen zwischen den Eigenlogiken nicht geleugnet und Bildungs- und Erfahrungsräume geschaffen werden, in denen ihr Widerstreit im 
Akzeptiertes Manuskript in: Karidi, M. u.a. (Hg.): Resilienz. Wiesbaden: Springer 2018, 203-225

Rahmen einer jeden Praxis ausgetragen und offen sowie öffentlich thematisiert werden kann (vgl. ebd., S. 31).

\subsubsection{Multimodalität von Resilienz}

Im Kontext systemisch-ökologischer Ansätze werden drei Weisen von Resilienz unterschieden, nämlich Persistenz (Standhaftigkeit), Adaption (Anpassung) und Transformation (Wandel) (vgl. z.B. Vogt und Schneider 2016b, S. 187f.). Diesen deskriptiven Modi stellen Schneider und Vogt drei normative Leitbilder gegenüber, nämlich Selbsterhaltung, Kontrolle und Lernen (vgl. in diesem Band). Die Persistenz von Strukturen und Systemen zielt auf Maßnahmen der "Gefahrenabwehr und Risikominimierung“ und damit auf „Selbsterhaltung“ (Vogt und Schneider, 2016b, S. 188). Adaption dient auch der Selbsterhaltung und meint die „Fähigkeit, ,im Einklang mit einem sich schnell wandelnden Umfeld zu ko-existieren““ (zitiert nach ebd.). Transformation zielt auf die „Fähigkeit, neue Strukturen und Systeme zu schaffen, weil die vorhandenen nicht mehr tragfähig sind“ (ebd.). In Bildungsprozessen sind damit prinzipiell auch als normative Zielperspektiven drei Modi relevant: a) Abwehr von Gefahren, Risiken usw. b) Anpassung an die sich ändernden Verhältnisse und c) Transformation von gesellschaftlichen u.a. Systemen und Strukturen.

\subsubsection{Multifaktorialität und Habitualisierung}

Die psychologische Resilienzforschung versucht psychische Ressourcen zu identifizierten, die als protektive Faktoren benannt werden sollen. Angeführt werden beispielsweise: Kohärenzgefühl, Selbstwahrnehmung, Selbstwirksamkeit, Selbstwert (vgl. z.B. Sautermeister 2016, S. 218), Religiosität u.v.a.m. In Ratgeberbüchern werden solche, teils stark differierende Listen, vorgestellt. Von wissenschaftlicher Seite werden nicht nur die Ratgeberbücher, sondern auch entsprechende Listen „kritisch gesehen, weil Resilienz entscheidend von der Wechselwirkung, Intensität und Dauer der multiplen Risiken sowie von der individuellen Sensibilität für bestimmte Belastungsfaktoren abhängt“ (Vogt und Schneider 2016b, S. 183). In Bildungsprozessen sind nicht lediglich einzelne Faktoren anzustreben, sondern multiple, faktorielle Wechselwirkungen sind mit und durch die Teilnehmenden bildend zu bearbeiten. Darüber hinaus geht es nicht lediglich um ein Wissen möglicherweise resilienzförderlicher Handlungen, sondern um verinnerlichte, habitualisierte 
Akzeptiertes Manuskript in: Karidi, M. u.a. (Hg.): Resilienz. Wiesbaden: Springer 2018, 203-225

Handlungen, damit nicht nur träges Wissen, sondern resilienzstärkende Handlungen im Bildungsprozess gelernt werden können.

$>\quad$ Habitus ist die grundgelegte und grundlegende, bewegliche Haltung (vgl. die Aristotelische Hexis), die den Zusammenhang der eigenen Lebensführung mit dem Repertoire an gesellschaftlich akzeptierten Haltungen ebenso hervorbringt, wie des eigenen Verhaltens in der Zeit (vgl. Wüschner 2016, 111; Riegger und Heil 2017a).

\subsubsection{Vielfalt der Bildungsorte bzw. Erfahrungsräume mit Resilienzrelevanz}

Resilienzsensible Bildung kann sich an unterschiedlichen Lernorten ereignen, welche unterschiedlichen Theorien bzw. Wissensformen und unterschiedlichen Prägungen (z.B. freiwillig oder zwangsweise) unterliegen. Das Bildungsverständnis kann - in Ergänzung zum Lernverständnis - die Kraft der Unterscheidung „wirksam [...] halten, und zwar vor allem im Interesse an der Autonomie der lernenden Subjekte und an ihrer Widerstandskraft gegen die Verwertung ihrer erlernten Kenntnisse und Fähigkeiten durch enge Zweckkalküle ökonomischer oder politischer Art" (Dressel 2017, 1.1). Die Begegnung mit entsprechenden Theorien und Prägungen beeinflusst mit, welche Gestalten resilienzsensibler Bildung möglich sind bzw. angestrebt werden können. $\mathrm{Ob}$ und wie aus Bildungsorten Erfahrungsräume werden, ist voraussetzungsreich. Deshalb ist es Aufgabe von Pädagogik und Didaktik, diese Begegnungen in Bezug auf unterschiedliche Bildungsorte zu reflektieren und gegebenenfalls zu ermöglichen.

Berücksichtigt man, dass nicht nur dort Resilienz gelernt wird, wo explizit Lernprozesse angestrebt werden, sind mindestens intentionale und nichtintentionale Prozesse an folgenden Lern- bzw. Bildungsorten zu unterscheiden: Familie, Kindergarten bzw. Kindertagesstätte mit Peers, Schule mit entsprechenden Unterrichtsfächern, die auch außerschulische Lern- bzw. Bildungsorte umfassen können (analog Hochschulen), Kommunen, überkommunale Bildungsarbeit, Medien und Öffentlichkeit. Die beiden eingangs skizzierten Fälle beziehen sich beispielhaft auf Schule und Hochschule.

\subsubsection{Strukturelemente}

In dem skizzierten allgemeinen Verständnis (3.1.1) ist Resilienz eine „ResponseStrategie“ (Adger 2000, S. 347, vgl. Schneider und Vogt in diesem Band). Alienitätstheoretisch tut sich mit der fremden Bedrohung eine Differenz zum Eigenen 
Akzeptiertes Manuskript in: Karidi, M. u.a. (Hg.): Resilienz. Wiesbaden: Springer 2018, 203-225

auf. Bei einer rein sachlichen Antwort (engl. answer) auf die Bedrohung, würde die Antwort exakt auf die Bedrohung passen, sodass Bedrohung und Antwort symmetrisch zueinander stünden und eine registrierbare Antwort die Bedrohung beheben würde (vgl. Waldenfels 2015a, S. 60). In Kontexten, in denen Resilienz als Response-Strategie aktiviert wird, dürfte diese Koinsidenz kaum der Fall sein. Von answer ist deshalb Antwort als response zu unterscheiden (vgl. zum Ganzen auch Kaspari 2010, S. 200-205). Ich skizziere das Grundverständnis von Waldenfels und bringe es zunächst mit resilienzsensibler Bildung und weiter unten mit Professioneller Simulation in Verbindung.

Responsivität bezieht sich nicht „auf spezielle Verhaltensweisen wie etwa das Erteilen einer Auskunft oder die Beantwortung einer Prüfungsfrage, sondern auf einen Grundzug“ (Waldenfels 2015b, S. 19) des Menschseins. Responsivität ist leiblich geprägt und kann „Hinsehen, Hinhören, Phantasieren, Lächeln oder Fühlen“ (ebd.) ebenso umfassen wie „Reden, Tun, Machen oder Herstellen“ (ebd.). Antworten (engl. to respond) in diesem Sinne bedeutet es grundlegend „auf Fremdes eingehen, das sich nicht mit den vorhandenen Mitteln des Eigenen und Gemeinsamen bewältigen läßt“ (ebd.). Antworten ist dann kreatives, schöpferisches Antworten. Diese Grundbedeutung weist offensichtliche Ähnlichkeiten mit Grundzügen von Resilienz auf, insofern auf Unerwartetes, Unbekanntes oder Ungewöhnliches mit Neuem reagiert wird. Wenn aber Resilienz die Person und ihre Widerstandskraft stärkt, so ist Responsivität nicht notwendig stärkend, denn sie kann für Resilienz bedeutungslos sein oder diese gar schwächen.

Eine Response erfolgt immer aufgrund eines „Widerfahrnisses als Getroffensein“ (Waldenfelds 2002, S. 56, H.i.O.). Widerfahrnis (gr. Pathos) meint, dass „uns etwas zustößt, zufällt, auffällt oder einfällt, dass uns etwas trifft, glückt oder auch verletzt“ (Waldenfels 2015b, S. 20). Pathos oder Widerfahrnis sind nicht einfach beobachtbare „Events“ (ebd.), da sie sich nur aus der Teilnehmerperspektive erschließen. „Was mir, dir, uns oder anderen zustößt, äußert sich in einer leibhaftigen Wirkung, indem es uns affiziert, wörtlich: antut oder anmacht, und indem es an uns appelliert, uns anspricht“ (ebd., S. 21). Die Ähnlichkeit zu Bildung ist offensichtlich: Sich bilden kann jede/r nur selbst, aber im Sinne Waldenfels präzise nicht als Autor, sondern als „Patient: ,Mir stößt etwas zu“, ,Mich hat etwas getroffen““ (ebd., S. 22, H.i.O.), „worauf" (ebd.) ich antworte. Im Waldenfelsschen Sinne heißt „Antworten [...] vom Fremden her sprechen. Damit verwandle ich mich vom 
Akzeptiertes Manuskript in: Karidi, M. u.a. (Hg.): Resilienz. Wiesbaden: Springer 2018, 203-225

Patienten in einen Respondenten, der auf das antwortet, was ihm widerfährt" (ebd., H.i.O.). Jeder Mensch muss antworten, weil er „nicht nicht antworten“ (ebd., H.i.O.) kann. Doch im „Antworten verwandelt sich das Worauf des Antwortens in das Was einer Antwort“ (ebd.). Ähnlich wie im Bildungsprozess sind bei Waldenfels der Akt des Subjektes (Waldenfels würde von Selbst sprechen) und die Sache zutiefst aufeinander verwiesen, wobei das Subjekt als Subjekt im Akt als grundlegend erachtet wird. Im Unterschied $\mathrm{zu}$ einem weitgehend outputorientierten Lernverständnis strebt Waldenfels eine klare Gewichtsverlagerung bzw. Umorientierung an (vgl. Waldenfels 2015a, S. 58): weg vom Antworten als reines Füllen von Wissenslücken, also ein reines Lernen von auswendig gewussten Inhalten als wiederholbares Antworten (answer) hin zu Responsivität. Auch das Was einer Antwort auf ein Widerfahrnis ist responsiv und damit immer kreativ, erfinderisch (vgl. Waldenfels 2015b, S. 23). Im Antworten entsteht aus der Subjektperspektive etwas Neues. Dieser schöpferische Prozess, der auch stocken oder misslingen kann, entwickelt sich aufgrund der Differenz „zwischen dem, worauf wir antworten und dem, was wir antworten“ (Waldenfels 2015a, S. 58), was als „responsive Differenz" (ebd.) bezeichnet wird.

Responsivität ohne Intentionalität ist nach Waldenfels undenkbar. Intentionalität meint zunächst, „dass sich etwas als etwas zeigt, dass etwas in einem bestimmten Sinn und in einer bestimmten Weise gemeint, gegeben, gedeutet, verstanden oder behandelt wird, nämlich als frisches Grün“ (Waldenfels 2015a, S. 34) usw. Gemeint ist, dass „etwas (Wirkliches, Mögliches oder auch Unmögliches) mit etwas (einem Sinn, einer Bedeutung) verbunden und zugleich von ihm geschieden ist“ (ebd., S. 34f.). In diesem anfänglichen Sinne erweist sich Intention als „Bedeutungsintention“ (Waldenfels 2002, S. 25). Doch ist etwas als etwas deuten nicht die ganze Sache, denn im „Bedeuten“ ist das „Begehren“ enthalten (vgl. Waldenfels 2002, S. 380). Begehren meint, „dass etwas, das fehlt oder abwesend ist, ,in etwas“ erstrebt wird“ (ebd.). Das erste Etwas ist nach Waldenfels das Woraufhin des Strebens oder das Gesamtstreben des Lebens (z.B. das Glücksstreben), während das zweite Etwas (in „in etwas“) die konkreten Sachen sind, die als Gegenstände der Begierde gedeutet werden. „Dieses appetitive In gehört ebenso wie das signifikative Als zu den Angelpunkten, um die sich die Erfahrung dreht“ (ebd. S. 380). Damit ist Intentionalität hauptsächlich durch das Ineinander der beiden Grundmechanismen Bedeuten (etwas als etwas: Sinn) und Begehren (etwas in etwas: Ziel) gekennzeichnet. Eine 
Akzeptiertes Manuskript in: Karidi, M. u.a. (Hg.): Resilienz. Wiesbaden: Springer 2018, 203-225

solche Intentionalität wird "durch die Responsivität nicht ersetzt, wohl aber überboten. Responsivität steht für eine ,Antwortlichkeit', die der Verantwortung für das, was wir tun und sagen, unwiderruflich vorauseilt" (Waldenfels 2015a, 57) und damit lebensstärkend ist.

$>$ Responsive Resilienz ist kreativ-schöpferisches Antworten auf Widerfahrnisse des Lebens mit lebensstärkendem Potential.

Vergleicht man das Waldenfelssche Denken mit Bildungsdiskursen, so müsste erstens verstärkt auf eine begriffliche Differenzierung von Intention und Ziel bzw. Kompetenz (vgl. Riegger 2005, S. 12) hingewiesen werden. Soll Resilienz nicht nur als kognitives Wissen angestrebt werden, sondern wirklich wirksam an Bedeutung gewinnen können, so sind zweitens in resilienzsensiblen Bildungsprozessen nicht nur abfragbare Kompetenzziele, sondern auf das Leben der Bildungsteilnehmenden bezogene Intentionen zu berücksichtigen, die im Bildungsprozess notwendig überboten werden.

\subsection{Professionelle Simulation und responsive Resilienz}

\subsubsection{Begründungsfiguren für resilienzsensible Bildung}

Wenn Bildung die Menschen stärkt, indem sie ,die Sachen klärt' (vgl. 2), konvergieren Bildung und Resilienz offensichtlich. Eine resilienzsensible Bildung gründet nicht nur auf den individuellen Prinzipien Bildsamkeit des Menschen und Aufforderung zur Selbsttätigkeit, sondern berücksichtigt auch Transformationen im gesellschaftlichen Bereich, sodass Resilienz in Bildungsprozessen nicht einseitig auf Personen und Persönlichkeitsbildung bezogen werden darf (vgl. 2).

Wenn Bildung zudem nicht nur dort wirksam werden kann, wo resilienzsensible Bildungsprozesse ziel- bzw. kompetenzorientiert geplant und durchgeführt werden, sondern auf das Leben beziehbarer Sinn mitberücksichtigt werden muss, so ist auch dies noch keine Gewähr dafür, dass Resilienz tatsächlich bildend relevant wird, da Resilienz als Response-Strategie Intentionalität immer auch überbietet. In der pädagogisch-didaktischen Begründung der Planung von Bildungsprozessen ist damit auch das Unplanbare einzuplanen.

\subsubsection{Grundverständnis von Simulation}


Akzeptiertes Manuskript in: Karidi, M. u.a. (Hg.): Resilienz. Wiesbaden: Springer 2018, 203-225

Simulation ist ein Begriff, der in der Alltagssprache und im Wissenschaftskontext vorkommt. In der Alltagssprache ist er mit (Vor-)Täuschung zumeist negativ, aber in den Medien auch neutral konnotiert. Im Wissenschaftskontext wird Simulation mit spezifischen Bedeutungen verwendet. Simulation meint hier die Modellierung hochkomplexer, realer Systeme, um das Verhalten von Systemen und deren zugrundeliegende Ursachen besser verstehen und abschätzen zu können (vgl. Heil und Riegger 2017, S. 86). Es wird sowohl ein wirklichkeitsähnliches und komplexitätsreduziertes Modell der Wirklichkeit hergestellt, wie in der Anwendung mögliche Szenarien als Simulationsergebnisse produziert werden (ebd., S. 88).

Im Bildungsbereich können Simulationen auf wirklichkeitsähnliche und komplexitätsreduzierte Nachahmung und Vorwegnahme offensichtlich widerständiger, wirklicher Situationen zielen (ebd., S. 93) (vgl. das zweite, eingangs skizzierte Fallbeispiel). Darüber hinaus können sich aber Simulationen auch auf mehr oder weniger alltägliche Widerfahrnisse beziehen. Erst in der bildenden Bearbeitung dieser Situationen können das Leben stärkende Aspekte entdeckt werden. Resilienz kann dann in der Auseinandersetzung mit den zuvor von den Teilnehmenden modellierten Situationen thematisch werden. Ein Unterschied zum pädagogischen Rollenspiel ist dabei der habituelle Bezug, der nicht nur zu Beginn, sondern durchgängig vorliegt, um habituell verankerte Kompetenzen, hier Resilienzkompetenzen, für einen bestimmten Bereich zu entwickeln.

Simulation ist eine „Als-ob“-Handlung in der Anwendung und Herstellung eines komplexitätsreduzierten und wirklichkeitsähnlichen Modells von Wirklichkeit zur Bildung habituell verankerter Kompetenzen in einem bestimmten Bereich (Heil und Riegger 2017, S. 96).

Resilienzsensible Bildung zielt damit auf Resilienzkompetenzen und erfolgt habituell, wenn mindestens Überzeugungen, Werthaltungen, Einstellungen, Motivationen und Selbstregulationen im Bildungsprozess thematisch werden.

\subsubsection{Phasen der Struktur der Professionellen Simulation}

Um die Struktur des Vorgehens herauszuarbeiten, das hinter den beiden eingangs skizzierten Fällen steht, erläutere ich - ohne weitere Reflexion des Bildungsprozesses - die erwähnten Phasen und bringe sie mit dem Responseverständnis in Verbindung (3.2.4). 
Akzeptiertes Manuskript in: Karidi, M. u.a. (Hg.): Resilienz. Wiesbaden: Springer 2018, 203-225

Szenendesign: Die Szene, welche den Ausgangspunkt der Simulation bildet, ist - der Wirklichkeit möglichst ähnlich nachgebildet - zu modellieren. Der Aufbau dieser Szene ist zentral, da der Aussagewert der Simulation wesentlich von den in dieser Ausgangsszene erfassten Ausgangsbedingungen abhängig ist.

Einstimmung durch die Leitung: Hier soll die Aufmerksamkeit aller Simulationsteilnehmenden aktiviert werden. Damit Subjekte und Inhalte zusammenkommen können, benötigt man Hinweise zum Kontext der Simulation, um die Ähnlichkeit zwischen Modell und äußerer Wirklichkeit - respektive Wirklichkeit zweiter und erster Ordnung - für alle nachvollziehbar darzustellen.

Impuls der Leitung: Ist alles für die Simulation vorbereitet, bedarf es eines Impulses (lat. pellere $=$ stoßen), um die Simulation in Gang zu setzen.

Handlungen durchführen: Nun wird die eigentliche Simulation durchgeführt. Simuliert werden beispielsweise vergangene Erlebnisse beim Frühstück oder unterschiedliche Handlungsalternativen für den Fall Mareike.

Kommunikation über eigene Erlebnisse: Diese Simulationsperspektive gibt dem Simulierenden die Gelegenheit, seine eigenen Wahrnehmungen auszudrücken und das selbst Erlebte sich selbst und anderen explizit zu vergegenwärtigen. Die subjektive, einer externen Beobachtung nicht zugängliche Perspektive, wird durch das Erzählen öffentlich, für andere einseh- und - mehr oder weniger nachvollziehbar.

Perspektivenwechsel: Die Kommunikation über die Erlebnisse anderer ist hier leitend. Um Grenzüberschreitungen zu vermeiden, können vorher geklärte Feedbackregeln hilfreich sein. Die Beobachtenden bringen sich ein, indem sie ihre Perspektive aus ihrer Rolle heraus darstellen.

Beendigung der Simulation: Die simulierten Rollen werden komplett abgelegt, die Szene abgebaut, die benutzten Utensilien beiseitegestellt. Danach beginnen Gespräche über die gesamte Simulation.

Habitusreflexion: In der Reflexion werden explizite Bewertungen des simulierten Erlebens angestellt. Die Reflexion erfolgt in mindestens zweifacher Entfaltung: Reflexionen bezogen auf den pragmatisch-reflexiven Habitus ebenso wie auf den reflexiv-wissenschaftlichen. Ggf. erfolgt eine Reflexion in Bezug auf den professionsbiografisch-reflexiven Habitus.

Evaluation: Abschließend ist der Wirkung der Simulation nachzugehen. 
Akzeptiertes Manuskript in: Karidi, M. u.a. (Hg.): Resilienz. Wiesbaden: Springer 2018, 203-225

\subsubsection{Kon- und Divergenzen zwischen Professioneller Simulation (=ProfiS) und Resilienz als Response-Strategie}

Professionelle Simulation beginnt, wie Response, mit dem Angesprochensein durch Fremdes, das auch emotional treffen soll. Während Widerfahrnisse des wirklichen Lebens uns ungeplant treffen, muss die Leitung der Simulation, so es resilienzsensible Bildung sein soll, die Ausgangsszene begründet auswählen und verantwortungsvoll gestalten, damit die Teilnehmenden sich auf den Prozess einlassen können.

Auf das Widerfahrnis wird kreativ geantwortet, in den beiden skizzierten Fällen mit unterschiedlichen Frühstücksszenarien bzw. Handlungsmöglichkeiten gegenüber der Unterrichtsstörung. $\mathrm{Ob}$ eine solche Response resilienzförderlich ist, muss im Bildungsprozess herausgearbeitet werden. Damit gilt: Nicht jede resilienzförderliche Response-Strategie ist Ergebnis gestalteter Bildungsprozesse, aber eine spezifische Form von Bildungsprozessen (ProfiS) ist strukturell resilienzsensibel angelegt.

Während Waldenfels den Menschen grundsätzlich als homo respondense (2015b, S. 15) versteht, kann man ProfiS im Rahmen von Bildungsprozessen auf ein solches Verständnis beziehen, aber ProfiS überlässt es den Teilnehmenden, ob sie für sich resilienzförderliche Antworten finden und diese außerhalb des Bildungsprozesses anwenden.

\section{Potenzial resilienzsensibler Bildung}

Abschließend bündle ich meine Überlegungen in Bezug auf zukünftige resilienzsensible Bildung mit fünf übergreifenden Aspekten und fünf Thesen.

\subsection{Relevanz}

Da Resilienz sich durch ein umfassendes Verständnis auszeichnet, das inhaltlich meist offen und dennoch stark subjekt- bzw. situationsbezogen ist, fallen viele inhaltlich stark vorstrukturierende Lehr-Lernmethoden aus. Neben Simulation sind sicher weitere Methoden im Blick auf Resilienzsensibilität zu untersuchen. Diese Methoden müssen nicht nur dem dynamischen Verständnis von Resilienz Rechnung tragen, sondern auch über rein kognitive Wissensvermittlung hinaus den ganzen Menschen berühren können. Die obigen Ausführungen legen nahe, dass Professionelle Simulation einen Weg darstellt, resilienzförderlich und habitusbildend zu wirken. 
Akzeptiertes Manuskript in: Karidi, M. u.a. (Hg.): Resilienz. Wiesbaden: Springer 2018, 203-225

\subsection{Aktivierung}

Wenn Lehren und Lernen innerhalb von Bildungsprozessen zuweilen als einseitig kognitiv und aktivierungsschwach wahrgenommen wird, wird dem mit resilienzsensibler Bildung entgegengewirkt. Denn in solchen Bildungsprozessen werden Eigen- und Selbsttätigkeit angeregt, nicht nur kognitiv, sondern auch habituell, und damit auch auf die Wirklichkeit außerhalb von Bildungsprozessen bezogen. Dem damit angesprochenen Zusammenhang von sogenannter Praxis und Theorie ist weiter nachzugehen.

\subsection{Wirklichkeitsbezug}

Resilienzsensible Bildungsprozesse werden immer in Bezug auf außerhalb des Bildungsprozesses liegende Wirklichkeit gestaltet. Insofern die Teilnehmenden ihrerseits Anknüpfungen an ihre Wirklichkeit außerhalb des Bildungsprozesses herstellen, erfolgt ein Wirklichkeitsbezug über den Habitus der Teilnehmenden. In der Gegenwart des Bildungsprozesses wird auf vergangene Wirklichkeit Bezug genommen, damit Teilnehmende sich habituell für Zukünftiges rüsten können. Weil die Zukunft aber offen ist, müssen unterschiedliche resilienzförderliche Möglichkeiten erarbeitet und auf ihre Tragfähigkeit sowie bildende Kraft hin reflektiert werden.

\subsection{Bildsamkeit}

Nur einem bildsamen Menschen wird Resilienz zu Teil. Diese Aussage unterstreicht, dass der Mensch keine inm von Natur aus vorgegebene Bestimmung und Resilienz hat. Vielmehr verweist die Bildsamkeit des Menschen auf die immer schon gegebene Notwendigkeit, sich in Bildungsprozessen im Wechselbezug zu sich, zu anderen und der Welt zu bilden. So bringt der Mensch seine Bestimmung und damit verbunden auch Resilienz selbst hervor. Für Gläubige ist dieser Prozess von Gott umfangen und zuinnerst getragen. Der skizzierte Bildungsvorgang verschärft sich sicher aufgrund neuer, gesellschaftlicher, kultureller sowie religiöser und anderer Herausforderungen. Deshalb bedürfen resilienzsensible Bildungsprozesse einer umfassenden Reflexion, um normativen Vorstellungen nicht unbewusst zu erliegen. Weil Bildungsprozesse beim Subjekt einsetzen, sind gesellschaftliche Transformationen zu berücksichtigen, und zwar so, dass Resilienz nicht nur in Bezug auf einzelne Menschen, sondern auch im Hinblick auf Systeme reflektiert und bewertet wird. 
Akzeptiertes Manuskript in: Karidi, M. u.a. (Hg.): Resilienz. Wiesbaden: Springer 2018, 203-225

\subsection{Thematische Profilierung}

Die dargestellten Fallbeispiele bezogen sich aufs Frühstück von Schülerinnen und Schülern sowie den Umgang mit Unterrichtsstörungen im Rahmen der Lehrerbildung. Der erlebte Widerstand im Klassenzimmer und die Reflexion möglicher Bewältigungsmuster sind offensichtlich auf Resilienzförderung bezogen. Mit dem ersten Fallbeispiel wurde deutlich, dass Resilienz als Response-Strategie auch auf Themen beziehbar ist, die erst auf den zweiten Blick resilienzförderlich sind. Denn es scheint unbestritten, dass ein gemeinsames und gesundes Frühstück nicht nur die Lebensqualität der Schülerinnen und Schüler hebt, sondern insgesamt lebensstärkend sein kann. Deshalb sind weitere Themen resilienzsensibler Bildung auszuarbeiten.

\subsection{Thesen}

1. Resilienzsensible Bildungsprozesse stärken in der Außeinandersetzung mit Inhalten Menschen und Systeme.

2. Bildung kann immer nur resilienzsensibel geplant und durchgeführt werden, da

a) die Teilnehmenden selbst über konkrete Resilienzförderlichkeit urteilen und

b) Nichtintentionales für diese resilienzförderlich werden kann.

3. Resilienzsensible Bildung berücksichtigt nicht nur die Persönlichkeit der Teilnehmenden, sondern ebenso gesellschaftliche, ökologische u.a. Kontexte und wird damit in drei Modi relevant:

a) Abwehr von Gefahren, Risiken usw.,

b) Anpassung an die sich ändernden Verhältnisse und

c) Transformationen von gesellschaftlichen und anderen Systemen.

4. Resilienzsensible Bildung konkretisiert sich theoretisch als Response-Strategie, welche praktisch mit der Methode der Professionellen Simulation (ProfiS) umgesetzt und anhand der zeitlichen Verlaufsschritte bzw. Strukturelemente derselben prozessgesteuert ist.

5. Konkrete Wirkungen solcher Bildungsprozesse sind zwar nicht replizierbar, aber im Blick auf Resilienz so zu evaluieren, dass zukünftige, resilienzsensible Bildungsprozesse empirisch informiert gestaltet werden können. 
Akzeptiertes Manuskript in: Karidi, M. u.a. (Hg.): Resilienz. Wiesbaden: Springer 2018, 203-225

Adger, W. N. (2000). Social and ecological resilience. Are they related? Progress in Human Geography 24, 347-364.

Benner, D. (2014). Bildung und Religion. Nur einem bildsamen Wesen kann ein Gott sich offenbaren. Paderborn: Ferdinand Schöningh.

Boschki, R. (2011). Einführung in die Religionspädagogik. 2. Aufl. Darmstadt: wbg.

Bucher, G., Domsgen, M. (2016). Empowerment in religionspädagogischer Perspektive. Zeitschrift für Theologie und Kirche 33, S. 407-439.

Dörpinghaus, A., Poenitsch, A., Wigger, L. (2012). Einführung in die Theorie der Bildung. 4. Aufl. Darmstadt: wbg.

Dressel, B. (2017). Bildungstheorie. In: Wissenschaftlich-Religionspädagogisches Lexikon im Internet (WiReLex). https://www.bibelwissenschaft.de/stichwort/100224/. Zugegriffen: 15.05.2017.

Endreß, M., Maurer, A. (Hrsg.) (2015). Resilienz im Sozialen. Wiesbaden: VS-Verlag. Endreß, M. Rampp, B. (2015). Resilienz als Perspektive auf gesellschaftliche Prozesse. Auf dem Weg zu einer soziologischen Theorie. In: Ders., Maurer, A. (Hrsg.). (2015), Resilienz im Sozialen (S. 33-55). Wiesbaden: VS-Verlag.

Fröhlich-Gildhoff, K., Dörner, T., Rönnau-Böse, M. (2016). Prävention und Resilienzförderung in Kindertageseinrichtungen - PRiK. Ein Förderprogramm. 3. Aufl. München: Reinhardt.

Fröschl, M. (2015). Verwundet reifen. Salutogenes und Resilienz als hoffnungsvolle Zukunftsperspektiven. Stimmen der Zeit 234, S. 315-322.

Gärtner, J. (2016). „Lebensstark aus der Klage. Traditionen der Hebräischen Bibel in der Perspektive von Resilienz am Beispiel von Ps 22“, Praktische Theologie 51, S. 75-81.

Gennerich, C., Riegel, U. (2015). Wissenschaftstheorie. In: WissenschaftlichReligionspädagogisches Lexikon im Internet (WiReLex). https://www.bibelwissenschaft.de/stichwort/100001/. Zugegriffen: 15.05.2017. Göppel, R. G., Zander, M. (Hrsg.) (2017). Resilienz aus der Sicht der betroffenen Subjekte: Die autobiografische Perspektive. Weinheim u.a.: Beltz Juventa.

Hauschildt, E. (2016). Resilienz und Spiritual Care. Einsichten für die Aufgaben von Seelsorge und Diakonie - und für die Resilienzdebatten. Praktische Theologie 51, S. 100-105.

Heil, S., Riegger, M. (2017). Simulationsbasierte Kompetenzentwicklung. In: Dies. Der religionspädagogische Habitus. Professionalität und Kompetenzen entwickeln - 
Akzeptiertes Manuskript in: Karidi, M. u.a. (Hg.): Resilienz. Wiesbaden: Springer 2018, 203-225

mit innovativen Konzepten für Studium, Seminar und Beruf (S. 85-124). Würzburg: Echter.

Höfler, M. (2016). Bewältigungskapazität als Bildungsauftrag. Resilienz aus Perspektive der Erwachsenenbildung. In: Wink, R. (Hrsg.). Multidisziplinäre Perspektiven der Resilienzforschung (101-122). Wiesbaden: VS Verlag.

Kaspari, T. (2010). Das Eigene und das Fremde. Phänomenologische Grundlegung evangelischer Religionsdidaktik. Leipzig: Evangelische Verlagsanstalt.

Keul, H. (2016). Vulnerabilität und Resilienz. Münchener Theologische Zeitschrift 67, S. 224-233.

Klafki, W. (1991). Neue Studien zur Bildungstheorie und Didaktik. 2. Aufl. Weinheim: Beltz.

Kuhn, E. (2013). „Voll uncool: Frühstück vor dem Fernseher?!“ In: ASIS-Mitteilungen, München, S. 46-49.

Kumlehn, M. (2015). Bildung, religiöse. In: Wissenschaftlich-Religionspädagogisches Lexikon im Internet (WiReLex). https://www.bibelwissenschaft.de/stichwort/100082/. Zugegriffen: 15.05.2017.

Leipold, B. (2015). Resilienz im Erwachsenenalter. München: Ernst Reinhardt.

Leppert, K., Koch, B., Brähler, E., Strauß, B. (2008). Die Resilienzskala (RS) Überprüfung der Langform RS-25 und einer Kurzform RS-13. Klinische Diagnostik und Evaluation 1, 226-243

Nguyen, T. T., Bellehumeur, C., Malette, J. (2015). God Images and Resilieance. A Study of Vietnamese Immigrants. Journal of psychology and theology, 43, S. 271282.

Nida-Rümelin, J., Gutwald, R. (2016). Der philosophische Gehalt des Resilienzbegriffs. Normative Aspekte. Münchener Theologische Zeitschrift 67, S. 250-262.

Oser, F. (2013). Religionsunterricht als Stärkung religiöser Resilienz. Religionsunterricht an berufsbildenden Schulen, S. 10-12.

Oser, F., Gamboni, E., Düggeli, A., Maskonati, J. (2004). Die Zeitbombe des „dummen“ Schülers. Eine Interventionsstudie zur Erhöhung von Resilienz bei drohender und erfahrender Erwerbslosigkeit unqualifizierter Jugendlicher. Bern. http://www.snf.ch/SiteCollectionDocuments/nfp/nfp43_oser_synthesis13.pdf.

Zugegriffen: 15.05.2017. 
Akzeptiertes Manuskript in: Karidi, M. u.a. (Hg.): Resilienz. Wiesbaden: Springer 2018, 203-225

Pohl-Patalong, U., Richter, C. (Hrsg.) (2016). Resilienz - Problemanzeige und Sehnsuchtsbegriff. Praktische Theologie 51. Göttingen: Kaiser.

Richter, C. (Hrsg.) (2017). Ohnmacht und Angst aushalten. Kritik der Resilienz in Theologie und Philosophie. Stuttgart: Kohlhammer.

Richter, C., Blank, J. (2016). „Resilienz“ im Kontext von Kirche und Theologie. Eine kurze Einführung in den Stand der Forschung. Praktische Theologie 51, S. 69-74.

Riegger, M. (2017a). Der professionalisierte religionspädagogische Habitus. In: Heil, Stefan, Ders., Der religionspädagogische Habitus. (S. 33-62). Würzburg: Echter.

Riegger, M. (2017b). Bildung von Flüchtlingen. Interdisziplinäre Zusammenarbeit zwischen Praktischer Theologie und Sozialer Arbeit. ET-Studies (Journal of the European Society for Catholic Theology) 8, S. 139-159.

Riegger, M. (2016). Erfahrung. In: Wissenschaftlich-Religionspädagogisches Lexikon im Internet (WiReLex). https://www.bibelwissenschaft.de/stichwort/100193/. Zugegriffen: 15.05.2017.

Riegger, M. (2015). Formen didaktischen und methodischen Handelns. Lehrbrief 5 des Religionspädagogischen Kurses von Theologie im Fernkurs, hrsg. von der Katholischen Akademie Domschule Würzburg, 2. Aufl. Würzburg.

Riegger, M. (2012). Persönlichkeit von Menschen in pastoralen Berufen. Ein Beitrag zur Professionalisierung. Wege zum Menschen 64, 477-49.

Riegger, M. (2005). Planung und Gestaltung von Religionsunterricht konkret. Donauwörth: Auer.

Riegger, M., Heil, S. (2017a). Religionslehrerinnen- und Religionslehrerbildung als Habitusbildung. In: Dies., Der religionspädagogische Habitus. (S. 63-83). Würzburg: Echter.

Riegger, M., Heil, S. (2017b). Das religionspädagogische Potenzial von Simulation in der Religionslehrerinnen- und Religionslehrerbildung: Konzept und Fallbeispiele. In: Dies., Der religionspädagogische Habitus. (S. 125-163). Würzburg: Echter.

Rönnau-Böse, M., Fröhlich-Gildhoff, K. (2015). Resilienz und Resilienzförderung über die Lebensspanne. Stuttgart: Kohlhammer.

Sarubin, N. et. al. (2015). Erste Analyse der psychometrischen Eigenschaften und Struktur der deutschsprachigen 10- und 25-Item Version der Connor-Davidson Resilience Scale (CD-RISC). Zeitschrift für Gesundheitspsychologie 23, S. 112-122. DOI: $10.1026 / 0943-8149 / a 000142$. 
Akzeptiertes Manuskript in: Karidi, M. u.a. (Hg.): Resilienz. Wiesbaden: Springer 2018, 203-225

Sautermeister, J. (2016). Resilienz zwischen Selbstoptimierung und Identitätsbildung. Münchener Theologische Zeitschrift 67, S. 209-223.

Sajak, C. P. (2012). Einführung in die Religionspädagogik. In: Ders. (Hrsg.). Praktische Theologie, Theologie studieren - Modul 4 (65-119). Paderborn: Schöningh.

Schröder, B. (2012). Religionspädagogik. Tübingen: Mohr Siebeck.

Schneider, M., Vogt, M. (2016). Glaube, Hoffnung, Liebe als Resilienzfaktoren. Theologisch-ethische Erkundungen. Münchener Theologische Zeitschrift 67, S. 195208.

Schumacher, J., Leppert, K., Gunzelmann, T., Strauß, B., Brähler, E. (2005). Die Resilienzskala - Ein Fragebogen zur Erfassung der psychischen Widerstandsfähigkeit als Personmerkmal. (2005). Zeitschrift für Klinisch Psychologie und Psychiatrie 53, S. 16-39

Schweitzer, F. (2015). Religionspädagogik. In: WissenschaftlichReligionspädagogisches Lexikon im Internet (WiReLex). https://www.bibelwissenschaft.de/stichwort/100099/. Zugegriffen: 15.05.2017.

Sedmak, C. (2016). Konnektivität. Sozialethische Aspekte von Resilienz. Münchener Theologische Zeitschrift 67, S. 234-249.

Sedmak, C. (2013). Innerlichkeit und Kraft. Studie über epistemische Resilienz. Freiburg i.B.: Herder.

Stangl, E. D. (2016). Resilienz durch Glauben? Die Entwicklung psychischer Widerstandskraft bei Erwachsenen. Ostfildern: Grunewald.

Vogt, M., Schneider, M. (Hrsg.) (2016a). Theologische und ethische Dimensionen von Resilienz. Münchener Theologische Zeitschrift 67. St. Ottilien: EOS

Vogt, M., Schneider, M. (2016b). Zauberwort Resilienz. Analysen zum interdisziplinären Gehalt eines schillernden Begriffs. Münchener Theologische Zeitschrift 67, S. 180-194.

Vogt, M. (2009). Die Stärke der Schwachen. Vorstudien zu einem sozialethischen Programm. Münchener Theologische Zeitschrift 60, S. 2-17.

Wagnild, G. M.; Young, H. M. (1993). Development and psychometric evaluation of the Resilience Scale. Journal of Nursing Measurement 1, S. 165-178.

Waldenfels, B. (2002). Bruchlinien der Erfahrung. Phänomenologie, Psychoanalyse, Phänomenotechnik. Frankfurt a.M.: Suhrkamp. 
Akzeptiertes Manuskript in: Karidi, M. u.a. (Hg.): Resilienz. Wiesbaden: Springer 2018, 203-225

Waldenfels, B. (2015a). Grundmotive einer Phänomenologie des Fremden. 5. Aufl. Frankfurt a.M.: Suhrkamp.

Waldenfels, B. (2015b). Sozialität und Alterität. Modi sozialer Erfahrung. Frankfurt a.M.: Suhrkamp.

Welter-Enderlin, R., Hildenbrand, B. (Hrsg.) (2016). Resilienz - Gedeihen trotz widriger Umstände. 5. Aufl. Heidelberg: Carl-Auer.

Werner, E. E. (2011). Risiko und Resilienz im Leben von Kindern aus multiethnischen Familien. Ein Forschungsbericht. In: Zander, M. (Hrsg.), Handbuch Resilienzförderung (32-46). Wiesbaden: VS Verlag.

Werner, E. E. et. al. (1971). The children of Kauai. A Longitudinal Study from the Prenatal Period to Age Ten. Honolulu: University of Hawaii Press.

Werner, E. E., Smith, R. (1982). Vulnerable but invincible. A longitudinal study of resilient children and youth. New York: McGraw-Hill.

Wink, R. (Hrsg.) (2016). Multidisziplinäre Perspektiven der Resilienzforschung. Wiesbaden: VS Verlag.

Wustmann Seiler, D., Fthenakis, W. E. (2016) (Hrsg.). Resilienz. Widerstandsfähigkeit von Kindern in Tageseinrichtungen fördern. Beiträge zur Bildungsqualität. 6. Aufl. Berlin: Cornelsen.

Wüschner, P. (2016). Gegen-Eigentlichkeit. Haltung - Indifferenz - Einsamkeit. In: Kurbacher, F., ders. (Hrsg.): Was ist Haltung? Begriffsbestimmung, Positionen, Anschlüsse (109-128). Würzburg: Königshausen \& Neumann.

Zander, M. (Hrsg.) (2011). Handbuch Resilienzförderung. Wiesbaden: VS Verlag.

Ziebertz, H.-G. (2015). Gegenstand der Religionsdidaktik. In: Hilger, G., Leimgruber, S., Ders. Religionsdidaktik. Ein Leitfaden für Studium, Ausbildung und Beruf (18-28). 4. Aufl. München: Kösel.

Riegger, Manfred, *1967, Prof. Dr. theol., Dipl. Sozialpäd. (FH), studierte Katholische Theologie und Sozialwesen in Tübingen, Berkeley/CA und Benediktbeuern. Promotion, Habilitation, Lehrstuhl für Religionspädagogik und Didaktik des Religionsunterrichts an der Katholisch-Theologischen Fakultät der LudwigMaximilians-Universität München (Lehrstuhlvertretung), e-mail manfred.riegger@kaththeol.uni-muenchen.de. 